\title{
A CHARACTERIZATION OF OPERATORS MAPPING A CONE INTO ITS DUAL
}

\section{ALBERT NOVIKOFF ${ }^{1}$}

Introduction. Our point of departure is the following remark of Aronszajn and Smith, the $n$-dimensional case of a (real) Hilbert-space theorem contained in [1]. Let $A$ be a real symmetric nonsingular matrix, with associated quadratic form $(A x, x)$. A necessary and sufficient condition that the elements of $A^{-1}$ all be non-negative is that, to every $x \in R^{n}$ ( $R^{n}=$ the space of real $n$-tuples), there exists a corresponding $\tilde{x}$ with the properties (a) $\bar{x}_{i} \geqq\left|x_{i}\right|, i=1, \cdots, n$ and (b) $(A \tilde{x}, \tilde{x}) \leqq(A x, x)$.

If we introduce the orthant $P_{1}=\left\{x: x_{i} \geqq 0, i=1,2, \cdots, n\right\}$, then the remark states that a necessary and sufficient condition for $A^{-1} P_{1} \subseteq P_{1}$ is that, to each $x$ corresponds an $\tilde{x}$ satisfying (a) $\tilde{x} \pm x \in P_{1}$ and (b) $(A \tilde{x}, \tilde{x}) \leqq(A x, x)$. We give below a modification of the Aronszajn and Smith theorem, obtained by a modification of their proof whose essentials are already contained in [1]. The purpose of the modification is to replace the positive orthant $P_{1}$ by an arbitrary convex cone $P$. Indeed, the use of reproducing kernels in [1] needlessly restricts considerations in $n$-space there to cones with $n$ linearly independent generators. (With a suitable scalar product, and referred to a suitable basis, such a cone is essentially the non-negative orthant $P_{1}$ above.) By contrast, the theorem as formulated below exploits their considerations geometrically, without the use of reproducing kernels, extending its scope. In particular, the same remark holds for certain cones other than $P_{1}$ in $n$-space which have infinitely many generators, and for which the methods of [1] cannot apply directly. Throughout we use the notion of the dual $P^{*}$ of a convex cone $P: P^{*}=\{x:(y, x) \geqq 0$ all $y \in P\}$ or, more briefly, $P^{*}=\{x:(P, x) \geqq 0\}$.

Let $V$ be a real Hilbert space whose scalar product is written $(x, y)$ and let $P$ be a closed convex cone in $V$. We consider a map of $V$ onto itself by a nonsingular self-adjoint operator $B$ (that is, $(B x, y)$ $=(x, B y)$ for all $x$ and $y)$. The cone $P$ is mapped into the set $B P$, which is easily seen to again be a cone. How is the dual $(B P)^{*}$ of this new cone related to $P^{*}$, the dual of $P$ ? The answer is evident:

Presented to the Society, January 6, 1960 under the title Invariance of cones by positive definite operators; received by the editors February 28, 1964.

1 Currently on leave at the Courant Institute of Mathematical Sciences, New York University. 


$$
\begin{aligned}
(B P)^{*} & =\{x:(B P, x) \geqq 0\}=\{x:(P, B x) \geqq 0\} \\
& =\left\{x: B x \in P^{*}\right\}=B^{-1}\left(P^{*}\right) .
\end{aligned}
$$

(That is, $P^{*}$ transforms contragrediently.)

Let us say that a cone is obtuse if $P \supset P^{*}$, acute if $P \subset P^{*}$, and self$d$ ual if both inclusions hold. For example, the positive orthant in $R^{n}=\left\{x: x_{i} \geqq 0, i=1, \cdots, n\right\}$, is self-dual. The terminology is motivated by the fact that, in two dimensions, a cone is acute (obtuse) according to this definition if and only if its central angle is at most (at least) $\pi / 2$. For later convenience we formulate here

LEMma $0 . B P$ is obtuse iff $B^{-2} P^{*} C P$.

Proof. This is immediate from $(B P)^{*}=B^{-1} P^{*}$. We remark that the definition of $P^{*}$ and, hence, of obtuseness depend on the choice of scalar product.

We next introduce the ordering on $V$ induced by $P: x \geqq 0$ if and only if $x \in P$, and $x \geqq y$ if and only if $x-y \geqq 0$. The analogue of the condition (a) of the introduction for cones $P$ other than the nonnegative orthant is $\tilde{x} \geqq \pm x$, or, equivalently, $\tilde{x} \pm x \in P$. We observe that this implies $\tilde{x} \geqq 0$, or, equivalently, $\tilde{x} \in P$.

Lemma 1. A sufficient condition that a closed convex cone $Q$ be obtuse with respect to a given scalar product is that, given $u \in V$, there exists $a \tilde{u}$ such that (a) $\tilde{u} \geqq \pm u$ (with respect to $Q$ ) and $(b)(\tilde{u}, \tilde{u}) \leqq(u, u)$.

REMARK. It is sufficient for such $\tilde{u}$ to exist only for $u \in Q^{*}$, as the proof below shows.

Proof. We must show $Q^{*} \subset Q$. Given any $u$ and a corresponding $\tilde{u}$, form $u^{+}=(\tilde{u}+u) / 2, u^{-}=(\tilde{u}-u) / 2$. Clearly, $u^{+} \geqq 0, u^{-} \geqq 0,\left(u^{+}, u^{-}\right)$ $=((\tilde{u}, \tilde{u})-(u, u)) / 4 \leqq 0$ and $\tilde{u}=u^{+}+u^{-}$. Let it now be assumed that (c) $u \in Q^{*}$. We will establish the desired result by showing (a), (b) and

(c) imply $u=\tilde{u}$, for we have already observed that (a) implies $\tilde{u} \in Q$. To begin with, $\left(u^{+}, u^{-}\right) \leqq 0$. Since $u^{+}, u^{-}$are in $Q$, while $u \in Q^{*}$, we have $\left(u, u^{-}\right) \geqq 0$. But $\left(u, u^{-}\right)=\left(u^{+}, u^{-}\right)-\left(u^{-}, u^{-}\right) \geqq 0$ implies, in virtue of the above inequalities, that $\left(u^{+}, u^{-}\right)=0$ and $-\left(u^{-}, u^{-}\right)=0$ since each is nonpositive and their sum non-negative. We conclude that $u^{-}=0$, and so $\tilde{u}=u$. Since $\tilde{u} \in Q$, we obtain $u \in Q$, i.e., $Q^{*} \subset Q$.

LEMMA 2. The sufficient conditions (a) and (b) stated in Lemma 1 are also necessary: given that $Q \supset Q^{*}$, then, for each $u \in V$, there exists a $\tilde{u}$ such that (a) $\tilde{u} \geqq \pm u$ and (b) $(\tilde{u}, \tilde{u}) \leqq(u, u)$.

Proof. Given that $Q$ is obtuse, and given an arbitrary vector $u$, we must find the corresponding $\tilde{u}$. Let $u_{+}=$orthogonal projection of $u$ 
on $Q=$ closest element of $Q$ to $u$; let $u_{-}=$orthogonal projection of $-u$ on $Q=-$ (orthogonal projection of $u$ on $-Q$ ).

We assert that $u_{+}-u \in Q^{*}$ and $u_{-}+u \in Q^{*}$. In fact, the statement $u_{+}-u \in Q^{*}$, together with $\left(u_{+}-u, u_{+}\right)=0$, is the analog, for cones, of the well-known orthogonality relation that characterizes projections of a subspace $M$ : the vector $u_{+}$of $Q$ minimizing $\|u-v\|^{2}$ for all choice of $v$ in $Q$ must make $\left\|u-\left(u_{+}+t v\right)\right\|^{2} \geqq\left\|u-u_{+}\right\|^{2}$ for arbitrary $v \in Q$ and $t \geqq 0$. Thus $\left\|u-u_{+}\right\|^{2}-2 t\left(u-u_{+}, v\right)+t^{2}\|v\|^{2} \geqq\left\|u-u_{+}\right\|^{2}$ for all $t \geqq 0$, and, therefore, $\left(u_{+}-u, v\right) \geqq 0$, i.e., $u_{+}-u \in Q^{*}$. If we examine the case $v=u_{+}$and $|t|$ small, we find, moreover, $\left(u-u_{+}, u_{+}\right)=0$. Similarly, $u_{-}+u \in Q^{*}$ and $\left(u_{-}+u, u_{-}\right)=0$. Thus, if we let $\tilde{u}=u_{+}+u_{-}$, we have $\tilde{u} \pm u \in Q^{*}+Q \subset Q$, which is condition (a). We need only verify (b): $(\tilde{u}, \tilde{u}) \leqq(u, u)$. However, we recall $\|v \pm w\|\left\|^{2}=\right\| v\left\|^{2}+\right\| w \|^{2}$ if $(v, w)=0$. In particular,

$$
\begin{aligned}
& \left\|u_{+}-\frac{u}{2}\right\|^{2}=\left\|\frac{u_{+}}{2}+\frac{u_{+}-u}{2}\right\|^{2}=\left\|\frac{u}{2}\right\|^{2}, \\
& \left\|u_{-}+\frac{u}{2}\right\|^{2}=\left\|\frac{u_{-}}{2}+\frac{u_{-}+u}{2}\right\|^{2}=\left\|\frac{u}{2}\right\|^{2},
\end{aligned}
$$

and, therefore, $\|\tilde{u}\|^{2}=\left\|u_{+}+u_{-}\right\|^{2}=\left\|u_{+}-u / 2+u_{-}+u / 2\right\|^{2}$ $\leqq(\|u\| / 2+\|u\| / 2)^{2}=\|u\|^{2}$.

TheOREM. A necessary and sufficient condition that a positive definite self-adjoint operator $A$ satisfy $A^{-1} P^{*} \subset P$ is that, for any $x$, there exists an $\tilde{x}$ such that (a) $\tilde{x} \geqq \pm x$ (i.e., $\tilde{x} \pm x \in P)$ and (b) $(A \tilde{x}, \tilde{x}) \leqq(A x, x)$.

Proof. Since $A=B B^{*}$ where $B$ itself is self-adjoint, we may write $A^{-1} P^{*} C P$ in the form $B^{-1} P^{*} C B P$. Letting $Q=B P$, the inclusion relation of the theorem becomes the condition that $Q$ be obtuse by Lemma 0 . Let $B x=u, B \tilde{x}=\tilde{x}$. The condition $\tilde{x} \geqq \pm x$ with respect to the ordering induced by $P$ is equivalent to $\tilde{u} \geqq \pm u$ with respect to the ordering induced by $Q=B P$. Also, $(A x, x)=(B x, B x)=(u, u)$ and $(A \tilde{x}, \tilde{x})=(\tilde{u}, \tilde{u})$. Thus the theorem is transferable, under the mapping by $B$, to a necessary and sufficient condition that the cone $Q$ be obtuse. The necessity and sufficiency are established in the two previous lemmas.

Corollary 1. If $P=P^{*}$, the above is a necessary and sufficient condition that $A^{-1} P \subset P$. If the cone $P$ is generated by $n$ independent vectors, then $P=P^{*}$ for any choice of scalar product rendering these vectors orthogonal. The theorem then is applicable to those $A$ which are positive definite and self-adjoint with respect to such a scalar product; in this 
form, the theorem reduces to the case discussed in [1], which considers, without further loss of generality, the case $V=R^{n}, P=$ positive orthant, and the usual scalar product. However, there are cones for which $P=P^{*}$ and yet are not orthants no matter what the choice of scalar product since they do not have a set of $n$ generators (for example, the Lorentz or right circular cone, $\left.\left\{x: x_{1} \geqq\left(x_{2}^{2}+x_{3}^{2}+\cdots+x_{n}^{2}\right)^{1 / 2}\right\}\right)$. For these cones the theorem generalizes the result in [1].

REMARKS. (1) In general, the theorem stated here is a modification rather than a generalization of that in [1], since $A^{-1} P^{*} \subset P$ neither implies nor is implied by $A^{-1} P \subset P$. However, it is interesting to observe that if $P$ is acute $\left(P \subset P^{*}\right)$, then $A^{-1} P^{*} \subset P \Rightarrow A^{-1} P^{*} \subset P^{*}$, while if $P$ is obtuse, $A^{-1} P \subset P \Rightarrow A^{-1} P^{*} \subset P$. Thus (a) and (b) are sufficient for $A^{-1} P^{*} \subset P^{*}$ and necessary for $A^{-1} P \subset P$ in the acute and obtuse cases, respectively.

(2) A closed convex cone $P$ in $V$ with vertex 0 determines a like cone $P_{M}=M \cap P$ in any closed subspace $M$ and the scalar product in $V$ restricts to a scalar product in $M$. Thus we can introduce a relativized notion of obtuseness for $P_{M}$. It is easy to see that the relative dual of $P_{M}$ contains $P^{*} \cap M$, and, therefore $P_{M}$ is always obtuse if $P$ is. The content of Lemmas 1 and 2 is that the obtuseness of $P$ is equivalent to the relative obtuseness of $P_{M}$ for those two-dimensional subspaces $M$ spanned by vectors $u_{+}$and $u_{-}$which arise by projecting an arbitrary $u$ on $P$ and $-P$. In particular, $a$ cone is obtuse if its two-dimensional sections with nonempty relative interior are all obtuse. I know no direct proof of this.

The author is grateful to acknowledge the kind encouragement and frequent stimulation of Professor S. Karlin of Stanford University.

\section{BIBLIOGRAPHY}

1. N. Aronszajn, and K. T. Smith, Characterization of positive reproducing kernels. Applications to Green's functions, Amer. J. Math. 79 (1957), 611-622.

Stanford Research Institute 